\title{
Una propuesta de adaptación del Marco Europeo para los ESL
}

\author{
Elizabeth Coelho ${ }^{1}$, Judith Oller ${ }^{2}$ y Josep M. Serra ${ }^{2}$ \\ ${ }^{1}$ Ontario Institute for Studies in Education, Universidad de Toronto, Canadá \\ ${ }^{2}$ Departament de Psicologia, Universitat de Girona, España
}

Artículo recibido el 5 de octubre de 2014, aceptado el 13 de noviembre de 2014, versión final el 2 de diciembre de 2014 DOI: http://dx.doi.org/10.5565/rev/jtl3.612

\section{Resumen}

En este artículo abordamos la evaluación del progreso lingüístico del alumnado inmigrante o minoritario que empieza a aprender la lengua de la escuela años después que sus compañeros nativos, ya sea catalán, castellano, u otra lengua del país o una lengua extranjera, como en las aulas de CLIL donde le lengua vehicular es el inglés o el francés, por ejemplo. Este alumnado (que llamamos ESL, estudiantes de una segunda lengua o de una lengua adicional para fines escolares) lleva una doble carga, aprendiendo de forma simultánea el contenido curricular y la lengua vehicular de la escuela. Es importante seguir el progreso de los ESL para saber cómo y cuánto adaptar el currículo para que éste sea alcanzable para los ESL de varios niveles de competencia lingüística, y para garantizar que estos alumnos sigan desarrollando sus habilidades lingüísticas hasta que lleguen al nivel que tienen sus compañeros de la misma edad.

Palabras clave: Marco Común Europeo de Referencia para las Lenguas, aprendizaje de lenguas, Español como Segunda Lengua, evaluación

\section{Resum}

En aquest article abordem l'avaluació del progrés lingüístic de l'alumnat immigrant o minoritari que comença a aprendre la llengua de l'escola anys després que els seus companys nadius, ja sigui català, castellà, o una altra llengua del país o una llengua estrangera, com a les aules de CLIL on li llengua vehicular és l'anglès o el francès, per exemple. Aquest alumnat (que anomenem ESL, estudiants d'una segona llengua o d'una llengua addicional per a fins escolars) porta una doble càrrega, aprenent de manera simultània el contingut curricular i la llengua vehicular de l'escola. És important seguir el progrés dels ESL per saber com i quant adaptar el currículum perquè aquest sigui assolible per als ESL de diversos nivells de competència lingüística, i per garantir que aquests alumnes segueixin desenvolupant les seves habilitats lingüístiques fins que arribin al nivell que tenen els seus companys de la mateixa edat.

Paraules clau: Marc Comú Europeu de Referència per a les Llengües, aprenentatge de llengües, Espanyol com a Segona Llengua, avaluació

\begin{abstract}
This article deals with the evaluation of the linguistic progress of immigrant or minority students who start learning the language of the school years after their native born peers; whether the language be Catalan, Castilian, or another language of the country or a foreign language, as in CLIL classrooms where they vehicular language is English or French, for example. These students (herein they will called SSL: Students of a Second Language or an additional language for school purposes) bear a double burden, simultaneously learning curriculum content and the vehicular language of the school. It is important to follow the progress of SSL to know how and when to adapt the curriculum so that it is achievable for SSL with various levels of language proficiency,
\end{abstract}


and to ensure that these students continue to develop their language skills until they reach the same level as their peers of the same age.

Keywords: Common European Framework of Reference for Languages, language learning, Spanish as a Second Language, evaluation

\section{Introducción}

En este artículo ${ }^{1}$ abordamos la evaluación del progreso lingüístico del alumnado inmigrante o minoritario que empieza a aprender la lengua de la escuela años después que sus compañeros nativos - ya sea catalán, castellano, u otra lengua del país o una lengua extranjera, ya sea catalán, castellano, u otra lengua del país o una lengua extranjera, como en las aulas de CLIL donde le lengua vehicular es el inglés o el francés, por ejemplo. Este alumnado (que llamamos ESL, estudiantes de una segunda lengua o de una lengua adicional para fines escolares) lleva una doble carga, aprendiendo de forma simultánea el contenido curricular y la lengua vehicular de la escuela. Es importante seguir el progreso de los ESL para saber cómo y cuánto adaptar el currículo para que éste sea alcanzable para los ESL de varios niveles de competencia lingüística, y para garantizar que estos alumnos sigan desarrollando sus habilidades lingüísticas hasta que lleguen al nivel que tienen sus compañeros de la misma edad.

Lo que haremos en este artículo es discutir, en términos muy generales, la utilidad del Marco Común Europeo de Referencia para las Lenguas (Council of Europe, 2001; utilizado en toda Europa para evaluar la adquisición de lenguas extranjeras) en la evaluación del progreso lingüístico de los ESL en edad escolar. Seguidamente propondremos el desarrollo de continuos lingüísticos ("developmental continuum” en inglés, o sea una serie de etapas del desarrollo lingüístico) a través de descriptores de rendimiento que sean adecuados para los estudiantes que están aprendiendo la lengua escolar, en base a la adaptación de algunos indicadores del marco común de referencia para las lenguas. Terminaremos con un resumen de cómo usar la información recopilada en estos continuos para planificar y diseñar la instrucción para los ESL con varios niveles de competencia en la lengua escolar. 


\section{La importancia de saber qué desarrollo lingüístico tienen los ESL y poder seguirlo}

La mayor parte de profesores de apoyo lingüístico suele trabajar con grupos muy heterogéneos a nivel de conocimiento de la segunda lengua. Lo mismo pasa con los profesores del aula ordinaria, que pueden trabajar simultáneamente con ESL de distintos niveles de competencia en la lengua de instrucción. En esta situación, se requiere un proceso continuado de evaluación del desarrollo lingüístico de los estudiantes para evaluar el punto de partida de cada alumno, determinar los objetivos de aprendizaje adecuados en función de su edad y nivel de competencia lingüística, adaptar las actividades de enseñanza y aprendizaje, y hacer un buen seguimiento de los progresos de este alumnado a lo largo del tiempo.

\section{¿Por qué no usar los mismos criterios de evaluación que se usan con el resto de estudiantes?}

La mayoría de profesores, al menos los de primaria, están muy acostumbrados a trabajar con los mismos criterios de evaluación del lenguaje y la lectoescritura que se usan con los alumnos que ya hablan la lengua de la escuela. Estos criterios pueden ser adecuados cuando se usan de forma apropiada pero en general no son útiles para los ESL, o al menos, no lo son hasta que este alumnado no se esté acercando al nivel de desarrollo lingüístico que sus compañeros, porque sus puntos de partida son muy distintos. El uso de criterios de evaluación diseñados para evaluar al alumnado que ha ido aprendiendo la lengua toda su vida puede informar al profesorado que los ESL tienen un rendimiento inferior al de sus compañeros (lo que es de esperar), y continuar ofreciendo esta información a lo largo de los años. Esta información no es muy útil porque los profesores no necesitan saber lo que los ESL no pueden hacer en comparación con los hablantes nativos, sino qué es lo que pueden hacer en la nueva lengua, para conocer cómo el estudiante está progresando en función de su punto de partida inicial. El uso de criterios de evaluación específicamente diseñados para evaluar el rendimiento de los ESL permite a los profesores comparar la trayectoria del desarrollo lingüístico de cada ESL con la trayectoria normal de adquisición de la L2, usando esta información para planificar las 
actividades didácticas con el objetivo de que el alumno alcance el nivel de dominio lingüístico que tienen los hablantes nativos dentro de los siguientes 5 o 6 años.

Es evidente que los profesores necesitan tener herramientas adecuadas para poder observar y evaluar la adquisición de la lengua de instrucción entre los ESL, y poder comprobar si están haciendo el progreso esperado, año por año, hacia conseguir un nivel de competencia lingüística comparable al de sus compañeros nativos de la misma edad. Esta información ayudará a los educadores a planificar, ajustar, y evaluar el programa de apoyo que reciba o necesite cada ESL.

\section{EI Marco Común Europeo de Referencia para las Lenguas: una visión general}

Se han elaborado varios continuos para la evaluación del desarrollo lingüístico de niños y niñas en edad escolar, con descriptores y ejemplos del nivel de ejecución lingüística que normalmente se demuestran en cada nivel o etapa de desarrollo. Pero había poca coherencia entre los distintos continuos, lo que dificultaba hacer el seguimiento del progreso de los estudiantes que habían pasado de una escuela a otra o para saber exactamente cómo se tenía que adaptar la enseñanza para un estudiante específico.

Para superar este problema, el Consejo de Europa, gracias a un proceso de trabajo colaborativo entre los profesores de lengua y los investigadores de varios países, elaboró el Marco Común Europeo de Referencia para las lenguas (MCER, 2001). El Marco Europeo es un instrumento muy usado por la mayoría de profesores de lengua en Europa ${ }^{2}$. El modelo consta de seis niveles de competencia lingüística, que van desde el nivel de usuario básico hasta el de un usuario altamente competente en la lengua, cuyo rendimiento es prácticamente indistinguible del nivel de ejecución de un hablante nativo. Consta también de una base de datos con descriptores mucho más detallados que pueden ser utilizados o adaptados para ilustrar cada nivel de competencia en distintos idiomas y contextos. En un contexto como el actual, dónde es frecuente que las personas se muevan entre los países para estudiar o para trabajar, el uso de este marco común ayuda a garantizar que los programas educativos y las evaluaciones de los resultados estén estandarizados. Así, por ejemplo, documentos tales como 
los resultados de los exámenes, los expedientes académicos y los certificados de estudios pueden ser comparables entre países.

Es evidente que el Marco Europeo fue diseñado pensando en el rendimiento de estudiantes adultos con estudios avanzados, tal y como se pone de manifiesto en la descripción de las distintas habilidades lingüísticas en cada nivel de competencia. Por ejemplo, un niño de cinco años puede tener un nivel muy alto de desarrollo lingüístico para su edad, pero las descripciones el nivel de competencia C2 no son apropiadas para definir las habilidades lingüísticas que se pueden llegar a tener a esta edad. No se puede esperar que ningún niño de cinco años sepa "reconstruir la información y los argumentos procedentes de diversas fuentes, ya sean en lengua hablada o escrita, y presentarlos de manera coherente y resumida”. Por lo tanto, pensamos que se requiere una adaptación importante de esta herramienta para los ESL en edad escolar.

El Marco Europeo es, por definición, una guía o un marco inicial. Y, en consecuencia, no es una lista de control detallada para la evaluación continuada del rendimiento lingüístico de cualquier ESL, sea cual sea su edad, la lengua meta, o el contexto; si bien es una base muy útil de referencia con la que empezar, y este es precisamente su propósito.

\section{Usar el Marco Europeo como modelo para la evaluación de la competencia en la segunda lengua con estudiantes en edad escolar}

El Marco Europeo puede usarse como base para desarrollar un continuo lingüístico y algunos descriptores de competencia lingüística más adecuados para los ESL que están aprendiendo la lengua de instrucción. A continuación proponemos algunos ejemplos de las adaptaciones que hacen falta, basándonos en los mismos seis niveles y la evaluación de las mismas categorías principales del rendimiento lingüístico que señala el Marco Europeo. Usar los mismos niveles de referencia permite a los profesores hacer un seguimiento de los progresos lingüísticos de los estudiantes a lo largo de los cursos escolares, a fin de asegurar un buen apoyo en todas las clases y comprobar que los ESL, vistos individualmente y como grupo, están progresando lingüística y académicamente a buen ritmo a lo largo de su escolarización. Sin embargo, a diferencia de los descriptores del Marco Europeo, las subcategorías que proponemos están directamente relacionadas con actividades comunicativas típicas del contexto escolar, y los 


\section{descriptores se limitan a las conductas que los profesores pueden observar en el aula ordinaria} y en las actividades extraescolares. La tabla 1 es un resumen gráfico de un continuum de desarrollo lingüístico para los ESL en edad escolar, basado en los seis niveles de rendimiento lingüístico del Marco Europeo de Referencia.

Tabla 1. Continuum de desarrollo lingüístico basado en los seis niveles de rendimiento lingüístico del MCER

\begin{tabular}{|c|c|c|}
\hline \multirow{2}{*}{ 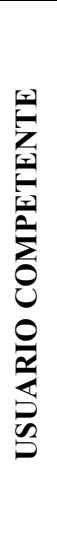 } & $\mathrm{C2}$ & $\begin{array}{l}\text { Los estudiantes pueden utilizar la segunda lengua de manera fluida y precisa en la mayoría de actividades } \\
\text { sociales y académicas, aunque la pronunciación y la entonación pueden verse influidos por la L1 en aquellos } \\
\text { estudiantes que empezaron a aprender la L } 2 \text { en el instituto. Los estudiantes pueden leer en la L2 materiales } \\
\text { apropiados para su edad con fines personales y académicos, utilizando estrategias de comprensión de manera } \\
\text { efectiva. Se comunican bien por escrito en una variedad de tareas con fines personales o académicos. Su } \\
\text { rendimiento lingüístico en la L2 es casi indistinguible del que tienen los hablantes nativos de la misma edad } \\
\text { que logran buenos resultados académicos. }\end{array}$ \\
\hline & C1 & $\begin{array}{l}\text { Los estudiantes usan la L2 con fluidez y confianza, comprendiendo la mayoría de detalles y vocabulario en } \\
\text { diversas asignaturas. Pueden leer una amplia gama de géneros textuales con fines personales y académicos, } \\
\text { utilizando estrategias efectivas de aprendizaje para manejar palabras nuevas, pero continúan necesitando algún } \\
\text { tipo de apoyo lingüístico en estructuras de oraciones complejas y cargadas de referencias culturales. Los } \\
\text { estudiantes pueden escribir en una variedad de géneros para propósitos y audiencias específicas, utilizando el } \\
\text { proceso de redacción de forma eficaz para corregir muchos de sus propios errores. Algunos errores } \\
\text { gramaticales ocasionales pueden pasar casi inadvertidos. }\end{array}$ \\
\hline \multirow{2}{*}{ 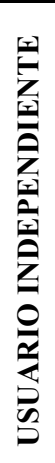 } & B2 & $\begin{array}{l}\text { Los estudiantes pueden comunicarse de forma efectiva en la mayoría de situaciones, utilizando gran variedad } \\
\text { de estrategias comunicativas, y pueden comprender informaciones detalladas sobre temas conocidos. Pueden } \\
\text { leer textos de estructura simple y algún material adecuado a su edad usando varias estrategias de comprensión } \\
\text { lectora. Los estudiantes pueden escribir textos usando un amplio repertorio de vocabulario en todas las áreas } \\
\text { curriculares con una precisión cada vez mayor, aunque los errores todavía son evidentes. }\end{array}$ \\
\hline & B1 & $\begin{array}{l}\text { Los estudiantes pueden iniciar interacciones y participar en los debates de clase, comprendiendo las ideas } \\
\text { principales y algunos detalles. Leen textos de estructura simple para su disfrute personal y para recopilar } \\
\text { información. Con apoyo del tutor empiezan a usar estrategias de comprensión. Pueden opinar sobre textos, } \\
\text { con andamiaje, como por ejemplo, los organizadores gráficos o párrafos incompletos proporcionados por el } \\
\text { profesor. Los estudiantes pueden escribir con fines personales y académicos, usando una amplia gama de } \\
\text { estructuras gramaticales, aunque los errores son todavía frecuentes. Con el apoyo del profesorado, empiezan a } \\
\text { usar el proceso de redacción para revisar su propio trabajo. }\end{array}$ \\
\hline \multirow{2}{*}{ 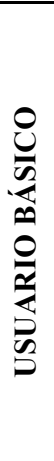 } & A2 & $\begin{array}{l}\text { Los estudiantes pueden comprender la segunda lengua cuando se les habla claro y despacio, iniciar y } \\
\text { responder a preguntas simples y participar en conversaciones breves. Los errores gramaticales y en la elección } \\
\text { de palabras son frecuentes y a veces impiden la comunicación. Pueden leer textos adaptados para los ESL de } \\
\text { este nivel para su diversión o para buscar información, y localizar las ideas principales del texto y algunos } \\
\text { detalles. Pueden escribir una serie de oraciones relacionadas en torno a una idea central con el apoyo de los } \\
\text { organizadores gráficos y utilizando un vocabulario familiar. }\end{array}$ \\
\hline & A1 & $\begin{array}{l}\text { Los estudiantes pueden usar palabras y frases simples para hacer o contestar preguntas, siempre que sus } \\
\text { interlocutores usen un habla lenta y cuidadosa. En lectura, primero abordan la comprensión del texto con la } \\
\text { ayuda de las ilustraciones y luego reconocen palabras frecuentes y leen frases comunes y oraciones sencillas } \\
\text { que aparecen en los materiales didácticos diseñados para los ESL de este nivel. En la escritura, los estudiantes } \\
\text { empiezan etiquetando u copiando palabras y frases, y progresan escribiendo oraciones simples para transmitir } \\
\text { información personal, usando imágenes y algunas palabras escritas. }\end{array}$ \\
\hline
\end{tabular}


En la tabla 2 se muestra como se pueden adaptar los niveles de referencia del MCER para los ESL en edad escolar.

Tabla 2. Adaptación a los ESL en edad escolar

\begin{tabular}{|c|c|c|c|c|c|c|}
\hline \multicolumn{7}{|c|}{ Ejemplo de tabla de 6 niveles para la evaluación del componente "Comprensión" del rendimiento lingüístico en la escuela } \\
\hline & A1 & A2 & B1 & B2 & C1 & $\mathrm{C2}$ \\
\hline 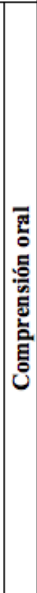 & $\begin{array}{l}\text { Comprende: } \\
\text { Palabras y frases muy } \\
\text { habituales acerca de } \\
\text { experiencias } \\
\text { personales y del } \\
\text { entorno inmediato } \\
\text { cuando se habla } \\
\text { despacio y con } \\
\text { claridad. }\end{array}$ & $\begin{array}{l}\text { Comprende: } \\
\text { Habla sencilla cuando se } \\
\text { utilizan frases y } \\
\text { vocabulario cotidianos } \\
\text { relacionados con temas } \\
\text { de su interés personal } \\
\text { (información personal y } \\
\text { familiar, lugar de } \\
\text { residencia, la escuela y } \\
\text { las rutinas de clase); } \\
\text { cuentos que se leen en } \\
\text { voz alta por el profesor, } \\
\text { siempre que el lenguaje } \\
\text { sea simple y haya un } \\
\text { apoyo visual; e } \\
\text { instrucciones y anuncios } \\
\text { de la escuela que sean } \\
\text { cortos y sencillos. }\end{array}$ & $\begin{array}{l}\text { Comprende: } \\
\text { La información esencial } \\
\text { que se explica en las } \\
\text { lecciones y actividades } \\
\text { de clase; textos cortos de } \\
\text { ficción o no-ficción que } \\
\text { se leen en voz alta por el } \\
\text { profesor; las ideas } \\
\text { principales transmitidas } \\
\text { por medios } \\
\text { audiovisuales en clase } \\
\text { cuando la articulación es } \\
\text { relativamente lenta y } \\
\text { clara, y cuando hay } \\
\text { pausas para discutir y } \\
\text { aclarar los segmentos de } \\
\text { información que no se } \\
\text { entiende; algunos } \\
\text { modismos y expresiones } \\
\text { coloquiales muy } \\
\text { comunes. }\end{array}$ & $\begin{array}{l}\text { Comprende: } \\
\text { El discurso extendido } \\
\text { del profesor y las } \\
\text { instrucciones de } \\
\text { múltiples pasos sobre } \\
\text { temas o actividades } \\
\text { conocidas; la mayoría de } \\
\text { los medios audiovisuales } \\
\text { usados en clase; el } \\
\text { material leído en voz } \\
\text { alta a velocidad normal, } \\
\text { siempre que el } \\
\text { vocabulario y las } \\
\text { expresiones utilizadas } \\
\text { hayan sido previamente } \\
\text { trabajadas; más } \\
\text { expresiones idiomáticas } \\
\text { y coloquiales. }\end{array}$ & $\begin{array}{l}\text { Comprende: } \\
\text { Con todo detalle el } \\
\text { discurso extendido de } \\
\text { las actividades } \\
\text { escolares, aunque } \\
\text { todavía pueden requerir } \\
\text { alguna repetición o } \\
\text { aclaración de vez en } \\
\text { cuando; los medios } \\
\text { audiovisuales utilizados } \\
\text { en clase sin mucho } \\
\text { esfuerzo aparente; } \\
\text { muchas expresiones } \\
\text { idiomáticas y } \\
\text { coloquiales. }\end{array}$ & $\begin{array}{l}\text { Comprende con } \\
\text { facilidad: } \\
\text { Todo lo que entiende la } \\
\text { mayoría de estudiantes } \\
\text { nativos de la misma } \\
\text { edad, ya sea en vivo, } \\
\text { grabado o retransmitido } \\
\text { por megafonia de la } \\
\text { escuela, aunque se } \\
\text { produzcan a una } \\
\text { velocidad de habla } \\
\text { estándar; hablantes con } \\
\text { distintos acentos; } \\
\text { lenguaje figurativo y } \\
\text { modismos, frases hechas } \\
\text { y expresiones } \\
\text { coloquiales usadas en } \\
\text { clase, dramatizaciones } \\
\text { teatrales y material } \\
\text { audiovisual usado en el } \\
\text { aula. }\end{array}$ \\
\hline 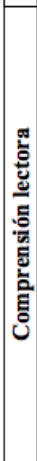 & $\begin{array}{l}\text { Comprende: } \\
\text { Nombres, palabras y } \\
\text { frases cotidianas muy } \\
\text { simples (por ejemplo, } \\
\text { pueden relacionar } \\
\text { palabras de la vida } \\
\text { cotidiana y de la } \\
\text { escuela con sus } \\
\text { respectivos dibujos); } \\
\text { historias en textos } \\
\text { simples e ilustrados } \\
\text { dirigidos a los ESL } \\
\text { principiantes. }\end{array}$ & $\begin{array}{l}\text { Comprende: } \\
\text { Textos cortos y simples, } \\
\text { diseñados para ESL con } \\
\text { un nivel básico de } \\
\text { conocimiento de la L2; } \\
\text { material cotidiano como } \\
\text { los anuncios } \\
\text { publicitarios, menús, } \\
\text { instrucciones y horarios } \\
\text { (por ejemplo, pueden } \\
\text { encontrar información } \\
\text { predecible y específica } \\
\text { con la ayuda del } \\
\text { profesor); cartas } \\
\text { personales simples. }\end{array}$ & $\begin{array}{l}\text { Comprende: } \\
\text { Textos escolares simples } \\
\text { que constan } \\
\text { mayoritariamente de } \\
\text { palabras frecuentes o } \\
\text { que hablen sobre temas } \\
\text { conocidos por el } \\
\text { estudiante, siempre que } \\
\text { se utilicen andamiajes } \\
\text { como organizadores } \\
\text { gráficos; lecturas } \\
\text { adaptadas para los ESL } \\
\text { de este nivel; artículos } \\
\text { cortos como los que se } \\
\text { encuentran en los } \\
\text { periódicos gratuitos. }\end{array}$ & $\begin{array}{l}\text { Comprende: } \\
\text { Noticias que salen en los } \\
\text { periódicos y algunas } \\
\text { novelas de ficción y no- } \\
\text { ficción apropiadas para } \\
\text { su edad, siempre que } \\
\text { algunas nuevas palabras, } \\
\text { expresiones, y } \\
\text { información cultural } \\
\text { que salen en el texto } \\
\text { hayan sido previamente } \\
\text { trabajadas; textos para } \\
\text { los ESL clasificados en } \\
\text { los niveles intermedio- } \\
\text { avanzado. }\end{array}$ & $\begin{array}{l}\text { Comprende: } \\
\text { Buena parte de las } \\
\text { novelas de ficción y no- } \\
\text { ficción apropiadas para } \\
\text { su edad, utilizando una } \\
\text { variedad de estrategias } \\
\text { de comprensión ante las } \\
\text { palabras desconocidas; } \\
\text { distintos géneros y } \\
\text { estilos de escritura, } \\
\text { siempre que el profesor } \\
\text { ofrezca modelos o } \\
\text { ejemplos. }\end{array}$ & $\begin{array}{l}\text { Comprende con } \\
\text { facilidad: } \\
\text { Todas las formas de } \\
\text { lengua escrita que se } \\
\text { esperan leer en la } \\
\text { escuela en función de la } \\
\text { edad, aunque en } \\
\text { ocasiones puedan } \\
\text { necesitar alguna ayuda } \\
\text { con el vocabulario o } \\
\text { algunas referencias } \\
\text { culturales. }\end{array}$ \\
\hline
\end{tabular}

La siguente tabla muesta una adaptación de la escala ilustrativa del componente "Monólogo sostenido: descripción de experiencias” para los ESL en edad escolar. 
Tabla 3. Muestra de sub-categorías

\begin{tabular}{|c|c|c|}
\hline \multicolumn{3}{|c|}{ Ejemplos de subcategorías para evaluar el progreso de los ESL en edad escolar } \\
\hline \multirow[b]{2}{*}{ 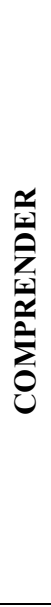 } & 范 & $\begin{array}{l}\text { - } \quad \text { Seguir instrucciones } \\
\text { - } \quad \text { Comprender presentaciones, explicaciones y lecturas en voz alta del profesor } \\
\text { - } \quad \text { Comprender la lengua oral mediante presentaciones audiovisuales } \\
\text { Comprender anuncios y otro tipo de información en audio }\end{array}$ \\
\hline & 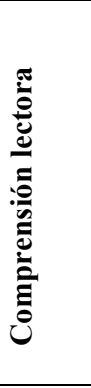 & $\begin{array}{l}\text { - Consciencia de la lengua escrita en la segunda lengua: por ejemplo, el alfabeto, o la dirección de } \\
\text { - } \quad \text { la escritura (para los estudiantes que no conocen el alfabeto latino) } \\
\text { - } \quad \text { analfabetos, o los ESL que saben leer pero que } \\
\text { - } \quad \text { Lectura independiente } \\
\text { - } \quad \text { Lectura de instrucciones } \\
\text { - } \quad \text { Lectura para la obtención de información } \\
\text { - } \quad \text { Estrategias de comprensión lectora } \\
\text { - } \quad \text { Opinar sobre la literatura }\end{array}$ \\
\hline \multirow[t]{2}{*}{$\frac{3}{3}$} & 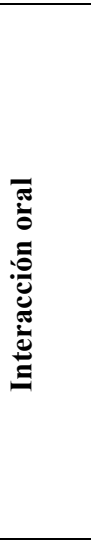 & $\begin{array}{l}\text { - } \quad \text { Expresión de necesidades } \\
\text { - } \quad \text { Comunicación no-verbal } \\
\text { - } \quad \text { Estrategias compensatorias (por ejemplo, sustituir otra palabra o una frase corta, o emplear } \\
\text { - } \quad \text { Estos o dibujos, para expresarse cuando no conoce la palabra exacta } \\
\text { - } \quad \text { Responder a preguntas } \\
\text { - } \quad \text { Participar en conversaciones } \\
\text { - } \quad \text { Vooperar en grupos } \\
\text { - } \quad \text { Uso del lenguaje coloquial, figurativo y de expresiones idiomáticas } \\
\text { - } \quad \text { Precisión gramatical } \\
\text { - } \quad \text { Conunciación de ideas } \\
\text { - } \quad \text { Uso de las reglas de cortesía y de registros formales e informales }\end{array}$ \\
\hline & 言 & $\begin{array}{l}\text { - } \quad \text { Hacer una presentación oral } \\
\text { - } \quad \text { Leer en voz alta }\end{array}$ \\
\hline$\underset{\substack{\mathscr{0} \\
\hdashline}}{\mathscr{0}}$ & 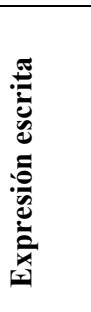 & $\begin{array}{l}\text { - } \quad \text { Convenciones de escritura en la segunda lengua (ortografía, puntuación, etc.) } \\
\text { - } \quad \text { Escritura personal y creativa } \\
\text { - } \quad \text { Escritura académica } \\
\text { - } \quad \text { Escribulario } \\
\text { - } \quad \text { Precisión gramatical } \\
\text { - } \quad \text { Formas de escritura y el uso del proceso de redacción } \\
\text { - } \quad \text { Uso de medios electrónicos y herramientas de referencia }\end{array}$ \\
\hline
\end{tabular}

Al utilizar tabla 3, profesores deben tener en cuenta que aunque la lengua se pueda categorizar de varias maneras, ésta se enseña, se usa y se aprende participando en las actividades del aula, algunas de las cuales requieren el uso integrado de las tres categorías de comunicación. También deben considerar que los ESL pueden mostrar distintos niveles de competencia en varios aspectos del uso de la lengua. Por ejemplo, un estudiante que ha estudiado la lengua en 
un país extranjero puede tener desarrollados niveles de lectura y escritura en la L2 más altos que en lengua oral, ya que las oportunidades que ha tenido para interactuar de forma oral en la nueva lengua han sido limitadas. Por otra parte, los estudiantes que han tenido una escolarización previa limitada pueden progresar mucho más rápidamente en el lenguaje oral que en la lectoescritura.

Los ESL pueden demostrar niveles de competencia lingüística distintos en función del contexto, según su conocimiento o experiencia previos: por ejemplo, puede que un estudiante con habilidades bien desarrolladas en la música sea capaz de comunicarse mejor en la clase de música que en una asignatura muy exigente lingüística y culturalmente, como las ciencias sociales o la literatura. Finalmente, se debe notar que "leer en voz alta" se incluye más bien como un aspecto de la expresión oral que de la comprensión lectora.

Tabla 4. Escala con descriptores para las "presentaciones de trabajos en el aula"

\begin{tabular}{|c|c|}
\hline \multicolumn{2}{|c|}{ Ejemplos de descriptores para la presentación de trabajos en el aula } \\
\hline $\mathbf{C 2}$ & $\begin{array}{l}\text { - Puede realizar presentaciones orales claras y fluidas, y a menudo destacables, sobre temas personales o } \\
\text { académicos, o presentar un punto de vista utilizando una variedad de soportes visuales y de medios electrónicos, } \\
\text { demostrando una plena consciencia de la audiencia a la que se dirige. } \\
\text { - Pocas veces necesita leer las notas durante la presentación. } \\
\text { - Algunos errores ocasionales pueden pasar casi inadvertidos. }\end{array}$ \\
\hline C1 & $\begin{array}{l}\text { - Puede realizar presentaciones claras y detalladas en el aula usando los medios apropiados. } \\
\text { - Utiliza las notas durante la exposición, pero mantiene el contacto visual con la audiencia. } \\
\text { Puede cometer errores gramaticales o en la elección de palabras de manera ocasional, pero no van en detrimento } \\
\text { de la comprensión. }\end{array}$ \\
\hline B2 & $\begin{array}{l}\text { - Puede realizar presentaciones claras y detalladas en el aula que hayan sido previamente ensayadas con un profesor } \\
\text { o mentor, utilizando los medios de comunicación y un lenguaje corporal apropiado. } \\
\text { - Depende de las notas para la exposición. } \\
\text { - } \quad \text { Comete errores evidentes y las auto-correcciones son frecuentes, pero no interfieren en la comprensión. }\end{array}$ \\
\hline B1 & $\begin{array}{l}\text { - Puede realizar descripciones o explicaciones sencillas relacionadas con el trabajo reciente hecho en clase. } \\
\text { - Puede narrar o relatar experiencias personales, acontecimientos recientes o historias personales, y describir } \\
\text { sueños, esperanzas y ambiciones usando palabras de enlace adecuadas, como, por ejemplo: después, hace algún } \\
\text { tiempo, etc. } \\
\text { - } \quad \text { Puede necesitar la ayuda de un guión de lectura previamente preparado para realizar la exposición; establece un } \\
\text { contacto visual limitado con la audiencia. } \\
\text { Comete frecuentes errores gramaticales o en la elección de palabras, y es capaz de corregir por sí mismo algunos } \\
\text { de ellos; los oyentes de vez en cuando pueden solicitar aclaraciones. }\end{array}$ \\
\hline
\end{tabular}




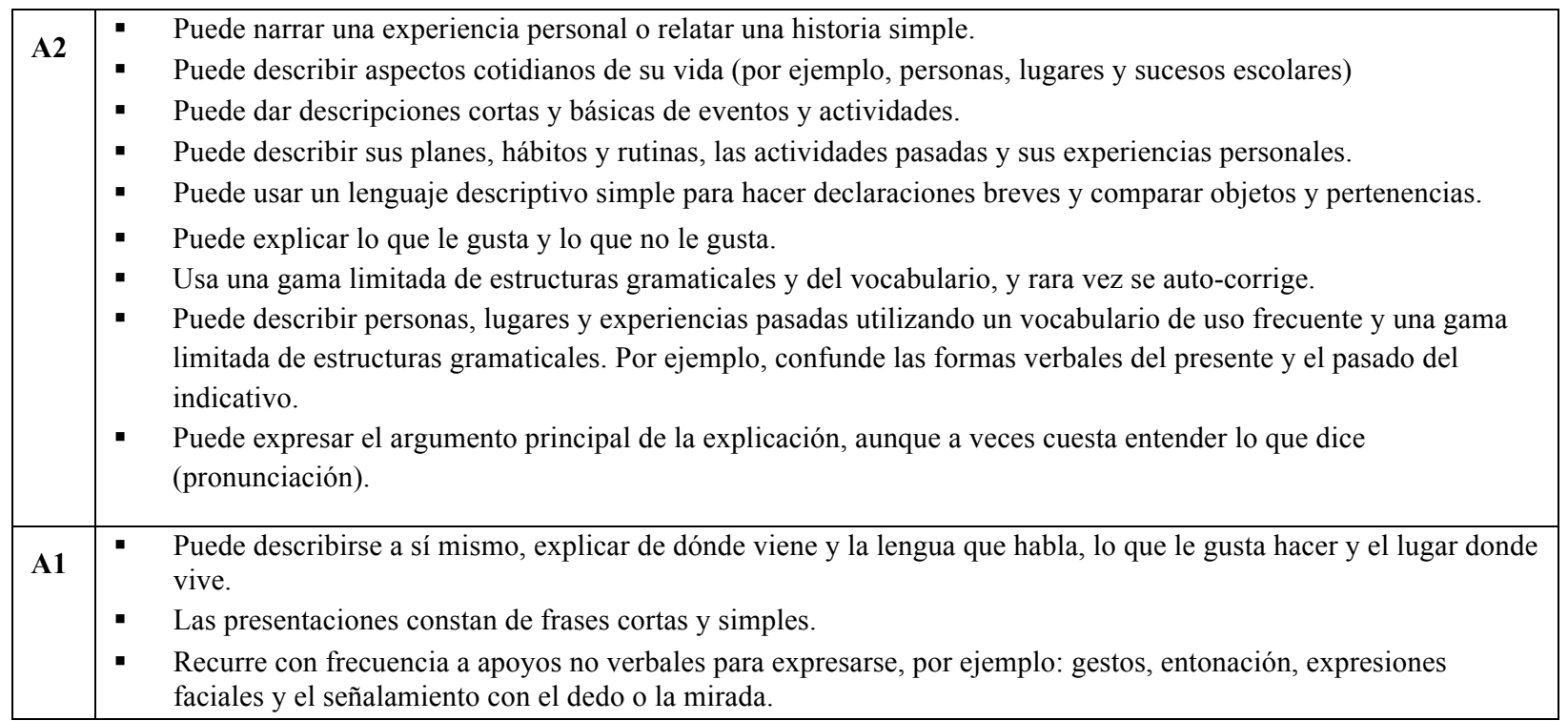

Los ejemplos de descriptores y escalas que hemos presentado en las páginas anteriores se han presentado como ejemplos de cómo hay que adaptar el Marco Europeo para elaborar continuos del desarrollo lingüístico con descriptores que se ajusten a las características de la L2 de cada comunidad autónoma o país, la edad de los ESL, su formación anterior, y el contexto educativo.

Queremos resaltar que hay que seguir continuamente el progreso lingüístico de cada ESL. Casi cada semana se pueden observar habilidades lingüísticas nuevas que no se vieron la semana anterior, ya que los aprendices de segundas lenguas están inmersos en un contexto ideal para el aprendizaje cuando están dentro del aula ordinaria (siempre y cuando la metodología de trabajo se adapte adecuadamente). Por ejemplo, los ESL de nueva incorporación en la escuela que al llegar no pueden demostrar tener ninguno de los descriptores de rendimiento para el nivel A1 de la lengua, a los pocos días o semanas de su escolarización en el nuevo país ya pueden empezar a mostrar algunos de ellos.

También es importante decir que usar un solo conjunto de descriptores para los estudiantes de cualquier edad no es adecuado, ya que los alumnos de distinta edad aprenden y utilizan la lengua de forma distinta, y también con propósitos distintos. Por esta razón, las subcategorías y los descriptores orientativos que presentamos pueden requerir de una mayor o menor adaptación en función de la edad de los grupos de ESL. Por ejemplo, en la educación infantil no se espera que un niño/a utilice la nueva lengua de la misma manera que lo hacen sus hermanos mayores, de ocho o catorce años. 
Otra cuestión a tener en cuenta es que los estudiantes que han tenido una escolarización previa irregular o interrumpida parten de un conocimiento en lectura y escritura muy distinto del que tienen otros compañeros de la misma edad que también son ESL pero que han recibido una enseñanza de lectoescritura en su propia lengua. Por otra parte, los estudiantes que hablan dialectos o variedades lingüísticas de la lengua de instrucción, como por ejemplo algunos estudiantes latinoamericanos que emigran a España, parten de niveles de competencia lingüística muy distintos de los que tienen otros compañeros cuya lengua familiar no coincide con la lengua de instrucción, a pesar que tampoco se pueden comparar con los hablantes nativos de la lengua estándar que se usa en la escuela. Es decir, que a pesar que no pueda parecerlo a simple vista, su progreso lingüístico va a ser distinto del que tienen los estudiantes nativos y del que tienen otros ESL.

En un contexto escolar, los profesores pueden utilizar continuos del desarrollo y descriptores de habilidades lingüísticas como una guía de observación del dominio de la lengua de instrucción que tienen los estudiantes en sus interacciones cotidianas dentro de la clase. Por ejemplo, utilizando la tabla anterior o una adaptación de ella en función de la edad de los alumnos, el profesor puede observar que, en una presentación oral de un trabajo el estudiante ha demostrado tener suficiente dominio de la lengua para "describirse a sí mismo, de dónde viene, la lengua que habla, lo que le gusta hacer y dónde vive" (uno de los descriptores del Nivel A1). El profesor puede marcar en la lista de control los descriptores que ha observado últimamente, tal vez usando una versión electrónica. Después esta información sirve como una guía para la planificación de las actividades que ayudarán al ESL a seguir desarrollando las mismas competencias lingüísticas a un nivel superior, o a empezar a desarrollar otras de este mismo nivel que aún no haya mostrado.

Toda la información que se recopile durante este tiempo y en los diversos contextos de aprendizaje puede ser útil tanto para los profesores, los directores y responsables de los consejos escolares, los estudiantes y sus familias, ofreciendo un perfil del progreso del estudiante y sus habilidades lingüísticas actuales. También es imprescindible para planificar la instrucción, tal y cómo sugerimos a continuación. 


\section{El modelo de los cuadrantes para la instrucción diferenciada}

Aprender una nueva lengua de instrucción es un proceso largo y costoso que no puede dejarse al azar. Según Cummins (2002), durante los dos primeros años de escolarización en una nueva lengua los ESL desarrollan habilidades comunicativas básicas o 'lenguaje cotidiano'. Al final de este período, la mayoría de estudiantes ya es capaz de desenvolverse con fluidez y soltura en las situaciones de comunicación informal más cotidianas. Sin embargo, el desarrollo de la lengua o 'lenguaje académico', necesita como mínimo 5 años para completarse. Durante todo este tiempo los ESL necesitan instrucción diferenciada, según a sus niveles de desarrollo lingüístico en el idioma escolar. Si no, van a perder mucho tiempo y dejarse más i más detrás: o bien porque a lección no está comprensible, o porque no es suficientemente exigente para que sigan desarrollando.

La figura 1 a continuación es una adaptación de un modelo propuesto por Cummins (2002) para diseñar la instrucción y la evaluación de los alumnos que están aprendiendo la lengua de la escuela, basado en las necesidades de los ESL y la cantidad de andamiaje (ayuda docente) necesarios para su progreso escolar. El modelo se estructura en cuatro cuadrantes creados a través de la intersección entre dos ejes.

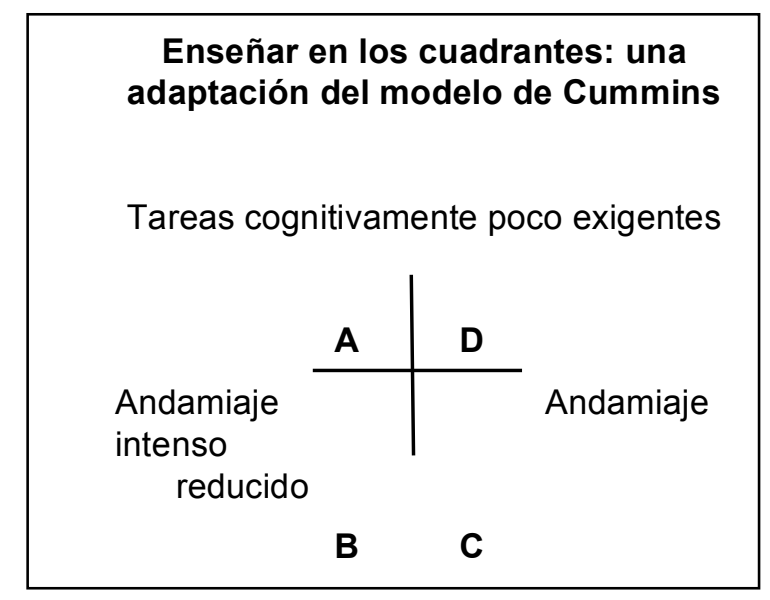

Figura 1. Modelo diseñar la instrucción y la evaluación de los ESL

En el eje horizontal, el continuum de andamiaje indica el grado de apoyo que se ofrece para ayudar a los ESL a comprender y a poder participar en las actividades que se realizan en el aula ordinaria a través de la lengua de instrucción. En el polo de "andamiaje intenso", en el extremo izquierdo del diagrama, los ESL aprenden la lengua dentro de un contexto significativo y familiar, dónde su comprensión es apoyada mediante la interacción cara a cara, las señales 
visuales, los ejemplos prácticos, la simplificación del lenguaje y otras pistas que les ayudan. En el extremo opuesto del eje, en cambio, el acceso al significado se realiza exclusivamente desde y a través del lenguaje.

En el eje vertical, la demanda cognitiva indica las exigencias de procesamiento de la información que requieren las actividades escolares. En el extremo "tareas cognitivamente poco exigentes", el lenguaje es simple y las actividades propuestas implican un bajo esfuerzo cognitivo por parte de los alumnos (por ejemplo, comprender y proporcionar información objetiva sobre un tema conocido). En cambio, en el extremo opuesto, el lenguaje que se utiliza para las actividades de enseñanza es complejo y las tareas de aprendizaje requieren que el alumno aprenda contenidos nuevos y movilice una gran variedad de procesos de pensamiento para poder manejar gran cantidad de información (por ejemplo, comparar, contrastar y evaluar diversas fuentes de información o puntos de vista).

\section{¿Cómo usar el modelo de los cuadrantes para diseñar la enseñanza?}

El siguiente diagrama muestra cómo las necesidades de los ESL van cambiando a medida que éstos van progresando a lo largo de las distintas etapas de desarrollo de la lengua de instrucción, con el objetivo final que puedan alcanzar el nivel de lenguaje académico que tienen sus compañeros nativos lo antes posible.

La instrucción para los principiantes tiene que iniciarse en el Cuadrante A, garantizando el máximo andamiaje para que puedan participar en actividades interesantes pero no muy exigentes cognitivamente. Gibbons (2009, p. 16) lo etiqueta "The Comfort Zone" (La zona de confort). Al principio, es probable que muy pocos objetivos del currículo ordinario sean alcanzables para los ESL recién llegados, y por eso deben sustituirse por objetivos lingüísticos relacionados con el lenguaje cotidiano para la comunicación básica. 


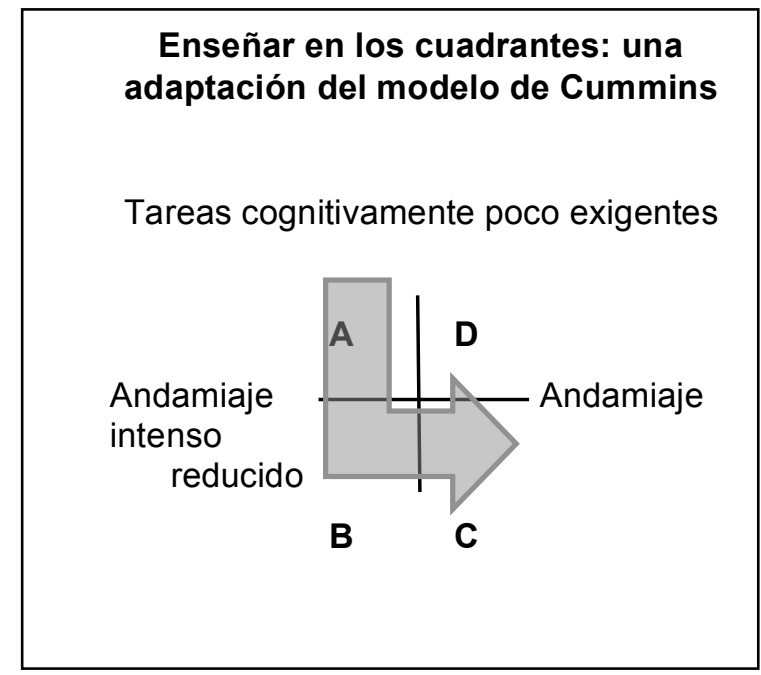

Figura 2. Necesidades cambiantes del alumnado ESL

Desde este punto de partida, y a medida que los ESL van demostrando más descriptores del nivel A1 y después del A2 (según un continuo de desarrollo adaptado del marco europeo para los ESL de edad escolar que están aprendiendo la lengua vehicular, tal y como ya hemos recomendado), las tareas y los objetivos se vuelven paulatinamente más exigentes, pero siempre alcanzables.

Una vez que los ESL demuestran haber alcanzado la mayoría de los descriptores del nivel A2, progresan al Cuadrante B, donde siguen recibiendo el máximo apoyo o andamiaje para realizar tareas y actividades escolares ya más exigentes, de forma que se empiece a acelerar la adquisición de su vocabulario académico. Gibbons lo etiqueta "The Learning/Engagement Zone" (La zona de aprendizaje e implicación”), basándose en la Zona de Desarrollo Próximo de Vygotsky (Gibbons, 2009: p. 16). Los ESL necesitan recibir instrucción en este cuadrante para poder abordar más objetivos del currículo ordinario, ya sea en una clase de apoyo lingüístico o bien dentro del aula ordinaria.

Una vez que los ESL demuestran haber desarrollado la mayoría de los descriptores del nivel A2, progresan al Cuadrante $C$ donde continúan trabajando en actividades cada vez más exigentes a nivel cognitivo, si bien el andamiaje del profesor va disminuyendo gradualmente en un período de entre 1 y 2 años. Cuando los ESL han podido alcanzar el nivel de competencia lingüística de sus compañeros de la misma edad, ya no necesitan más apoyo específico a parte del de mantener a todos los estudiantes implicados en las actividades de clase y progresando. Se puede hablar de "La zona de aumento de autonomía del estudiante". 
Es importante señalar que el progreso de la enseñanza en los cuadrantes no pasa por el Cuadrante D. En este cuadrante las actividades no son ni cognitivamente exigentes ni pueden incluirse en un contexto significativo. Los estudiantes, pues, no entienden el motivo de realizar este tipo de tareas. Es la "Zona de aburrimiento", ya que este tipo de actividades es poco probable que ayuden a mejorar la comprensión conceptual de los estudiantes o a promover el desarrollo del lenguaje académico. Si pensamos en lo que pasaba años antes, cuando las clases se limitaban a copiar lo que el profesor ponía en la pizarra y que no se comprendía, o bien a escuchar las lecciones magistrales que no siempre tenían sentido para nosotros, ese es el tipo de instrucción que se da en el cuadrante D, y ¡no debe producirse en ninguna aula!

El cuadro de la siguiente página proporciona un marco de referencia basado en el modelo de los cuadrantes para la instrucción diferenciada de los ESL en cada uno de los tres cuadrantes (A, B y C), así como una explicación más amplia de por qué el trabajo en el cuadrante D no es útil para la enseñanza y el aprendizaje.

Cuando se usa este marco como guía para la instrucción de los ESL, es importante ser flexible y sensible a las necesidades de estos estudiantes. Por ejemplo, puede haber estudiantes que necesitan instrucción en el cuadrante $\mathrm{B}$ en algunas áreas del currículo que son cultural y lingüísticamente exigentes, como las ciencias sociales, pero que pueden beneficiarse de la instrucción en el cuadrante $\mathrm{C}$ en otras materias que les sean más familiares para ellos, como las matemáticas o la música.

Es importante que los profesores sigan prestando apoyo lingüístico en el cuadrante $\mathrm{C}$, a pesar de que la mayoría de los ESL ya estén plenamente integrados en el aula ordinaria. Sólo de esta forma se puede asegurar que todos los ESL reciben el apoyo necesario para tener éxito en la escuela. Esto significa que todos los profesores, de quienes seguramente muy pocos hayan recibido una formación para adaptar la enseñanza a los ESL, tienen que hacer un esfuerzo para integrar la enseñanza de la lengua vehicular y sus estrategias de apoyo en todos los aspectos del currículo. Por lo tanto, los profesores especialistas en enseñanza de la lengua tienen que colaborar con sus compañeros para compartir estrategias y recursos que ayuden a adaptar el currículo en el aula ordinaria. El cuadro sobre el "andamiaje en los cuadrantes" (figura 3) puede servir de guía para iniciar esta colaboración. 
TAREAS COGNITIVAMENTE POCO EXIGENTES

\begin{tabular}{|c|c|c|c|}
\hline \multicolumn{4}{|c|}{ TAREAS COGNITIVAMENTE POCO EXIGENTES } \\
\hline 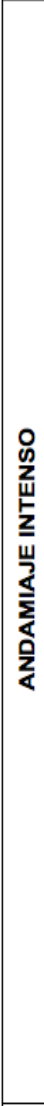 & 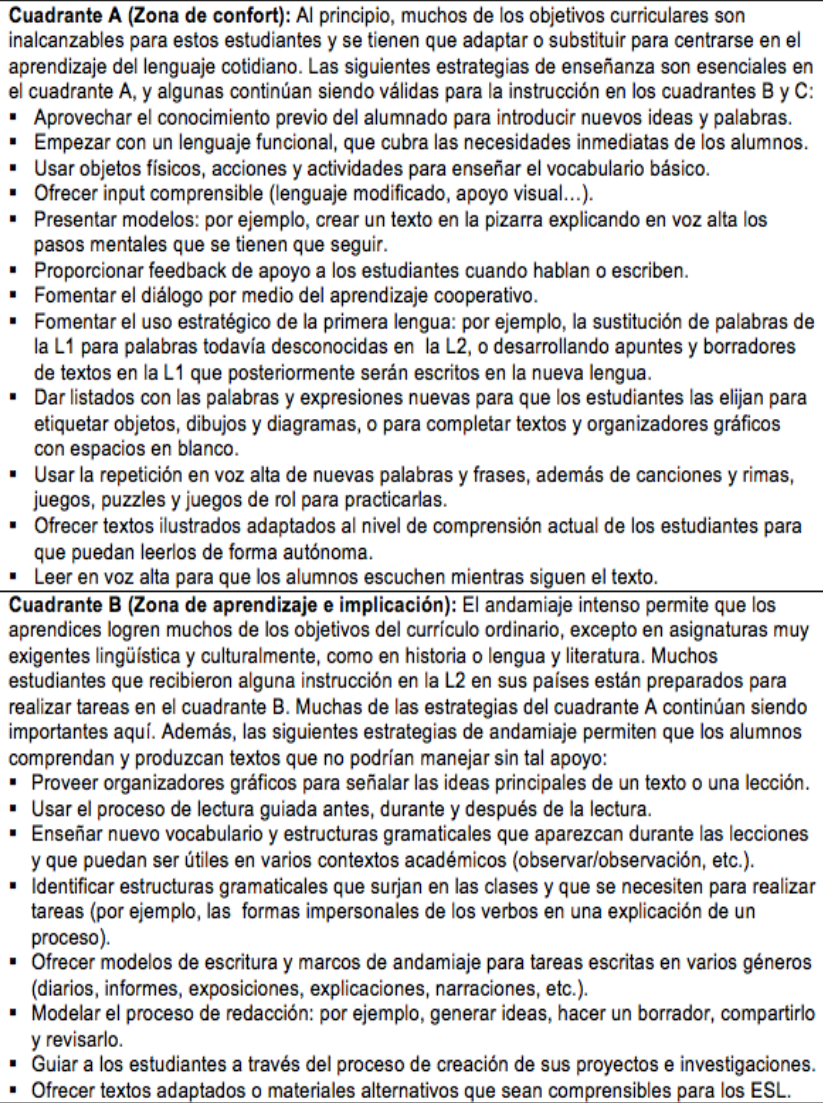 & 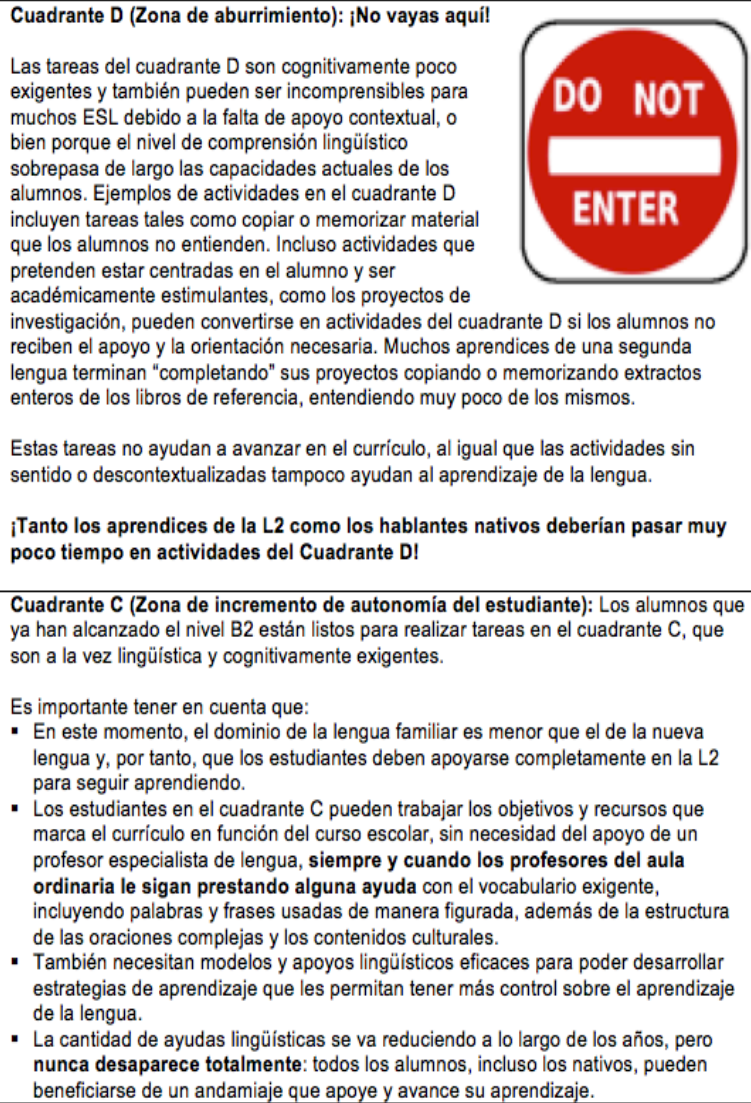 & 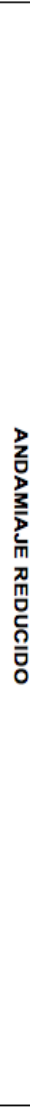 \\
\hline \multicolumn{4}{|c|}{ TAREAS COGNITIVAMENTE EXIGENTES } \\
\hline
\end{tabular}

Figura 3. Andamiaje en los cuadrantes: un marco para la instrucción diferenciada de los aprendices de segundas lenguas

\section{Conclusión}

Hemos destacado algunas características importantes del Marco Europeo, y hemos sugerido algunos ejemplos de cómo el marco se puede adaptar para elaborar unos continuos de desarrollo lingüístico aptos para alumnos de varias edades que estén aprendiendo la lengua vehicular como segunda lengua, o bien como lengua adicional. Dichos continuos se pueden utilizar para identificar los ESL y sus necesidades al incorporarse en la escuela. Además, se pueden utilizar para seguir detenidamente el progreso de los ESL a lo largo de la adquisición de los distintos descriptores, hasta que lleguen al Nivel C2. La información recopilada al seguir el progreso lingüístico de cada ELS se puede utilizar para planificar la enseñanza y para ofrecer un apoyo adecuado al nivel linguüístico de cada estudiante.

De esta forma, creemos que los educadores podrán asegurarse de que todos los ESL sigan desarrollando sus capacidades lingüísticas a un ritmo adecuado, y acabar alcanzando el nivel de competencia lingüística esperado en comparación con sus compañeros nativos de la misma edad. 


\section{Referencias}

Council of Europe. (2001). Common European Framework of Reference for Languages: Learning, Teaching, Assessment (CEFR). Cambridge, UK: Cambridge University Press. Se encuentra también en línea en varias lenguas (véase abajo).

Cummins, J. (2002). Lenguaje, poder y pedagogía. Madrid: MECD-Morata. Es un libro de lectura obligatoria para cualquier educador que enseñe en contextos multiculturales. Explica la distinción entre lenguaje conversacional y académico, y el tiempo que tardan los ESL a alcanzar el nivel de lenguaje académico que tienen sus compañeros nativos de la misma edad. También sugiere formas para aumentar el dominio lingüístico de los estudiantes.

Gibbons, P. (2009). English Learners, Academic Literacy, and Thinking. Portsmouth, NH: Heinemann. Estrategias prácticas para elevar la calidad de instrucción para los ESL, permitiéndoles desarrollar sus habilidades lingüísticas por actividades que los estimulan intelectualmente.

\section{Lectura adicional}

Ballano, I. (coord.) (2012). Evaluar la competencia lingüistica del alumnado inmigrante. Bilbao: Deusto Publicaciones. El libro incluye aportaciones de varios autores que reflexionan acerca de la evaluación de la competencia lingüística del alumnado extranjero y las dudas que ésta plantea, tanto desde un punto de vista técnico como en relación a las consecuencias que las acreditaciones de conocimiento de la lengua del país de acogida pueden tener para el inmigrante.

Roessingh, H., Kover, P., y Watt, D. (2005). Developing Cognitive Academic Language Proficiency: The Journey. TESL Canada Journal, 23 (1), 1-27. Los resultados de la investigación descrita en este artículo son muy útiles para el desarrollo de políticas y programas para los ESL, confirmando que estos estudiantes, incluso los que llegan a temprana edad, necesitan apoyo durante un largo tiempo para la adquisición de la L2.

\section{Páginas Web}

Council of Europe. Common European Framework of Reference for Languages (CEFR). Todos los materiales relacionados con el Marco Europeo. El marco mismo está disponible en todas las lenguas oficiales de España.

http://www.coe.int/t/dg4/linguistic/cadre1_en.asp

Cuaderno intercultural.org. Recursos de atención a la diversidad cultural en el contexto escolar. El sitio contiene información y enlaces interesantes sobre materiales de evaluación de las competencias iniciales del alumnado recién llegado.

http://www.cuadernointercultural.com

European Language Portfolio (ELP). Es un sitio de referencia para conocer toda la información sobre los cerca de 90 modelos de portafolio de evaluación para las lenguas de los 47 estados miembros pertenecientes al Consejo de Europa. La página Web incluye vídeos de presentación del portafolio europeo, ejemplos de portafolios elaborados en distintos países a través del Marco Europeo de Referencia, pruebas de evaluación de la competencia lingüística en varias lenguas, además de otra información 
general sobre proyectos lingüísticos en la Unión Europea. http://elp.ecml.at/ y también http://www.coe.int/t/dg4/education/elp/

METECO - Portal del español como segunda lengua en la escuela. Contiene enlaces en la parte de "evaluación", así como recursos para la enseñanza integrada de lengua y contenidos curriculares. http://meteco.ugr.es/

Rancé, L. (2005). Nivell inicial de català A1- usuari bàsic per a escolars de 8 a 14 anys. Barcelona: Departament d'Ensenyament. Generalitat de Catalunya. Material de referencia de la Generalitat de Cataluña para el profesorado que atiende a ESL en el que se explica la programación curricular y la evaluación del alumnado inmigrante recién llegado a Cataluña, adaptado al nivel A1 del Marco Europeo de Referencia. Disponible en: http://www.xtec.cat/crpbadalona/recursos/acollida0506/documentacio/nivell_inicial.pdf

${ }^{1}$ El presente artículo es una adaptación del Capítulo 3 del libro 'Lenguaje y aprendizaje en el aula multilingüe: un enfoque práctico' de Elizabeth Coelho, Judith Oller y Josep Maria Serra, publicado por ICE-Horsori en 2013. ${ }^{2}$ El Marco Europeo y otros materiales complementarios basados en éste pueden encontrarse en la Web del Consejo de Europa: www.coe.int/T/DG4/Linguistic/ . En España, el Instituto Cervantes ha hecho la traducción del Marco Común Europeo de Referencia y el documento íntegro puede descargarse desde la Web del Centro Virtual Cervantes (CVC): http://cvc.cervantes.es/obref/marco/. Además, algunas comunidades bilingües de España también se han preocupado en adaptar el Marco Europeo de Referencia a sus lenguas oficiales, como por ejemplo, en Cataluña.

\section{Referencias de los autores:}

Elizabeth Coelho ha sido profesora de inglés como segunda lengua/lengua extranjera en Inglaterra, Bulgaria, Canadá, y España. En la Universidad de Toronto trabajaba en la formación del profesorado, centrándose en la diversidad lingüística y cultural. Ha publicado varios libros y artículos en Canadá e Inglaterra, y últimamente en España.

Judith Oller es profesora asociada del Departamento de Psicología de la Universitat de Girona y consultora docente en la UOC. Sus intereses e investigación se centran en la educación bilingüe, la multialfabetización y la educación inclusiva. Tiene varias publicaciones relacionadas con estos temas.

Josep M. Serra es profesor titular del Departamento de Psicología de la Universitat de Girona. Sus intereses e investigación se relacionan con la organización y los resultados de la educación bilingüe, los programas de inmersión y las aulas de acogida. Ha publicado varios libros y artículos sobre estas temáticas.

Email (autora principal): e.coelho@utoronto.ca

Para citar este artículo:

Coelho, E., Oller, J. y Serra, J.M. (2015). Una propuesta de adaptación del Marco Europeo para poder adaptar la instrucción y seguir el desarrollo lingüístico de los aprendices de la lengua escolar. Bellaterra Journal of Teaching \& Learning Language \& Literature, 8(1), 10-27. http://dx.doi.org/10.5565/rev/jtl3.612 